\title{
Use of Primary Sutures to Stop Persistent Bleeding due to Hirudotherapy
}

\author{
Serkan Doğan', Mehmet Yiğit Özgün², Canan Akman', Utku Murat Kalafat', Tarık Ocak
}

'Department of Emergency Medicine, Kanuni Sultan Süleyman Education and Research Hospital, İstanbul, Turkey 2Department of General Surgeon, Kanuni Sultan Süleyman Education and Research Hospital, İstanbul, Turkey

\begin{abstract}
Introduction: The use of medicinal leeches for treatment purposes is known as hirudotherapy. Hirudotherapy has long been used in traditional medicine for the treatment of chronic pain, particularly in the back and knees. However, it may cause complications such as local infections, allergy, and continued bleeding. This report discusses the effectiveness of primary sutures in controlling continued bleeding caused by hirudotherapy in three patients who presented to the emergency department.

Case Report:Three patients presented to the emergency department because of bleeding in the region of where leeches were used for treating back pain. Vital signs of the patients were normal and have any diseases. Persistent bleeding stopped after primary suturing of the wounds.

Conclusion: Indications and contraindications of hirudotherapy should be identified in a detail by researches. Practitioners of this therapy should be informed of the use and removal of leeches to prevent the occurrence of complications.
\end{abstract}

Keywords: Back pain, bleeding, hirudotherapy, leech

Received: 17.11.2015 Accepted:05.01.2016 Available Online Date: 04.02.2016

\section{Introduction}

Treatment with medicinal leeches, known as hirudotherapy, has been used as a complementary and alternative medical method since ancient times $(1,2)$. Hirudotherapy is used as a treatment method globally, particularly in Far Eastern and Middle Eastern countries, for the treatment of chronic pain, sialadenitis, osteoarthritis, and venous insufficiency. However, there are side effects associated with this treatment such as continued bleeding, anemia, infection, and allergic reactions $(3,4)$. This report discusses emergency approaches and treatments in three cases that were presented to the emergency department after hirudotherapy for back pain.

\section{Case Report}

\section{Case 1}

A 33-year-old male patient was presented to the emergency department with continuous bleeding in his lumbar region. He reported that he used leeches for the treatment of back pain $24 \mathrm{~h}$ prior to presentation and that bleeding continued after this application. Anamnesis results indicated that he had not taken any drug therapy and that he did not have any chronic disease. Physical examination revealed seven leech bites measuring 2-3 $\mathrm{mm}$ on the lumbar region. The bites were bleeding in the form of a leakage, and there were ecchymotic lesions around the bites (Figure 1). The bleeding areas were dressed, and a pressure bandage was applied. His other physical examination revealed no abnormality. Blood tests performed to determine whether there was any bleeding diathesis revealed normal results. As the bleeding in the form of leakage did not cease after $2 \mathrm{~h}$ despite the application of a pressure bandage, primary sutures were made on the wounds in a sterile manner (Figure 2). After $2 \mathrm{~h}$ of follow-up, the bleeding ceased. We applied a tetanus vaccine to the patient. The patient was then prescribed antibiotics for infection control and was discharged from the hospital. He was asked to return for a control visit after 24 h. No complications 


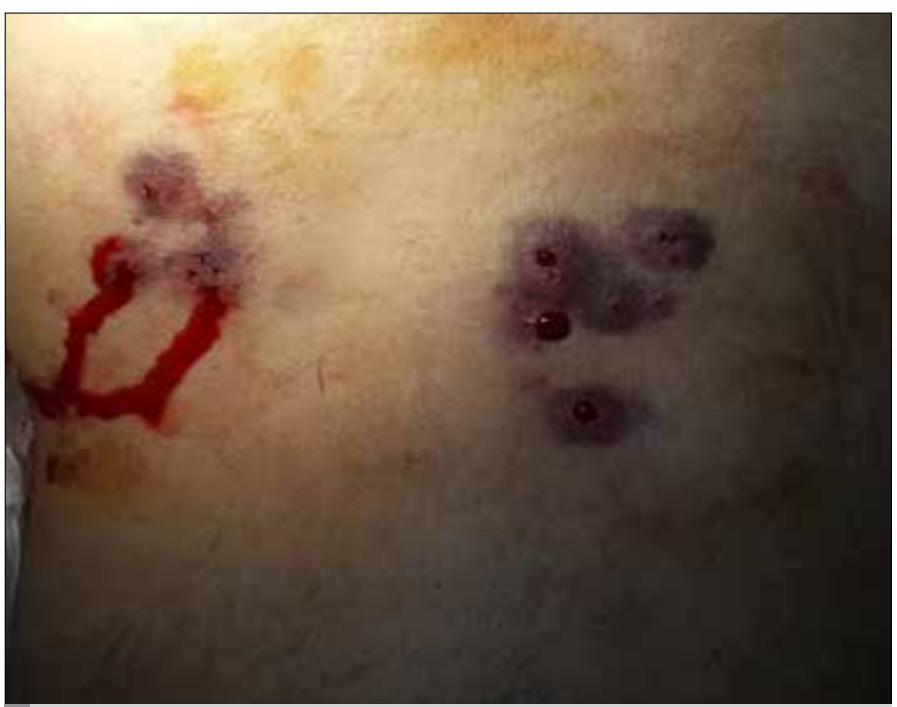

FIGURE 1. Bleeding in the form of a leakage

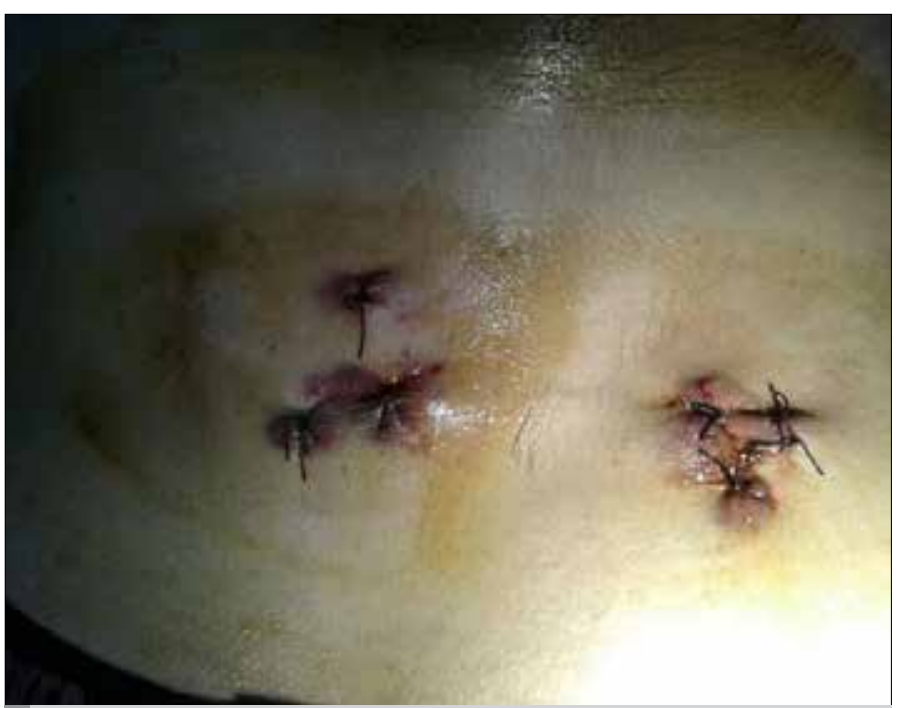

FIGURE 2. After primary suture

such as inflammation and bleeding were observed in the control examination.

\section{Case 2}

A 21-year-old male patient was presented to the emergency department because of persistent bleeding after hirudotherapy applied to the lumbar region. Upon the advice of his family, he reported that he had used leeches for the treatment of back pain $18 \mathrm{~h}$ prior to presentation. Physical examination showed eight leech bites measuring 2-3 $\mathrm{mm}$ on the lumbar region. The bites were bleeding in the form of a leakage. The bitten areas were dressed, and a pressure bandage was applied. His other physical examination revealed no abnormality. Blood tests performed to determine whether there was any bleeding diathesis revealed normal results. The bleeding did not cease after $2 \mathrm{~h}$ despite the application of a pressure bandage. The bleeding leech bites were closed with primary sutures. After $2 \mathrm{~h}$ of follow-up, the bleeding ceased. We applied a tetanus vaccine to the patient. The patient was then prescribed antibiotics for infection control and was discharged from the hospital. He was asked to return for a control visit after $24 \mathrm{~h}$. No complications such as inflammation and bleeding were observed in his control examination.

\section{Case 3}

A 35-year-old male used hirudotherapy for the treatment of pain in the lumbar region upon the advice of a friend. He applied leeches in the evening and removed them in the morning. Bleeding was seen in the form of leakage in the lumbar region after $10 \mathrm{~h}$ of contact with the leeches. On physical examination, five-six leech bites were seen on the lumbar region, which were bleeding in the form of a leakage. His other physical examination revealed no abnormality. After dressing, pressure tamponade was applied to the bites. Blood tests performed to check for bleeding diathesis revealed normal results. Because the bleeding did not cease after $2 \mathrm{~h}$ of pressure tamponade, the bleeding bites were closed using primary sutures. After 2 $\mathrm{h}$ of follow-up, the bleeding ceased. We applied a tetanus vaccine to the patient. The patient was then prescribed antibiotics for infection control and was discharged from the hospital. He was asked to return for a control visit after $24 \mathrm{~h}$. No complications such as inflammation and bleeding were observed in his control examination.

\section{Discussion}

Hirudotherapy is a traditional method in which leeches are used for the alleviation of chronic pain. In modern medicine, hirudotherapy is used to reduce venous congestion and to improve therapy outcomes particularly in plastic and reconstructive surgery (3). Moreover, hirudotherapy may complement medical drugs used for the alleviation of chronic pain associated with osteoarthritis in elderly patients (4). The three patients described in this study reported using leeches for the treatment of back pain.

Leeches are known to ingest ten times their body weight in blood. Leech secretion contains hirudin, a substance that inhibits thrombin, which plays a major role in the coagulation process. Furthermore, substances such as histamine secreted by leeches may cause continued bleeding by inhibiting the closure of capillaries and veins (5). Bleeding may be prolonged due to the collagen-thrombocyte interaction and the changes caused by proteases and other enzymes secreted by leeches during feeding. It is reported that bleeding resulting from leeches might last from $10 \mathrm{~h}$ to 7 days (6). At the end of treatment, saline solution or vinegar may be used to remove leeches from the skin. If they are removed forcibly, the possibility of bleeding or other undesirable side effects increases (7). In the cases discussed here, bleeding in the form of a leakage may have continued because the leeches were improperly removed.

In addition to continued bleeding, hirudotherapy is associated with the risk of the development of anemia, allergy, and anaphylaxis. Furthermore, there have been reports on the development of thrombotic microangiopathy and kidney failure after this type of therapy. Generally seen in the form of local abscess formation and cellulitis, infections also constitute significant complications of hirudotherapy (8). In the present study, all three cases had normal blood and kidney 
function test results, and no infections were detected in the leech bites. However, the rate of developing infection associated with hirudotherapy was reported to be between 2.4 and 4\%. This therapy must be performed in a sterile environment, and the use of appropriate antibiotics must be considered (7). For the cases described in this study, we planned appropriate antibiotic treatment regimens considering the antisepsis principles to prevent the development of infection.

Various methods of treatment have been suggested for long-term bleeding, which is a significant and common complication of medicinal leech therapy. One of the most common methods is the use of gauze bandages with solutions that prevent bleeding such as thrombin $(8,9)$. However, because such preparations are not readily available in all emergency departments, the number of cases in which only pressure bandages are used is greater. Electrocoagulation has been used successfully but is only practical in the hospital setting, and one case report demonstrated the use of hemostatic dressing for successfully ending leech wound bleeding (10). For the three cases in this study, although we applied pressure bandages for $2 \mathrm{~h}$, bleeding continued. Therefore, we decided to apply primary sutures, which yielded positive outcomes.

When laboratory test results are normal and the application of pressure bandages does not stop bleeding, primary sutures can be effective for controlling bleeding in the form of a leakage, as observed in this study (11).

\section{Conclusion}

Leech bites are a common occurrence worldwide, although rarely life-threatening. These wounds may bleed persistently from hours to days. We believe that emergency physicians should be aware of unstoppable bleeding due to leeches and should use primary sutures in such cases.

Informed Consent: Written informed consent was obtained from patient who participated in this case.
Peer-review: Externally peer-reviewed.

Author Contributions: Concept - S.D., M.Y.Ö.; Design - S.D., C.A.; Supervision - U.M.K., T.O.; Materials - S.D., M.Y.Ö., C.A.; Data Collection and/or Processing - S.D., M.Y.Ö., C.A.; Analysis and/or Interpretation - U.M.K., T.O.; Literature Review - S.D., U.M.K., T.O.; Writer - S.D., M.Y.Ö., U.M.K.; Critical Review - S.D., T.O.

Conflict of Interest: The authors declared no conflict of interest.

Financial Disclosure: The authors declared that this study has received no financial support.

\section{References}

1. Munshi Y, Ara I, Rafique H, Ahmad Z. Leeching in the history- a review. Pak J Biol Sci 2008; 11: 1650-3. [CrossRef]

2. Papavramidou N, Christopoulou-Aletra H. Medicinal use of leeches in the texts of ancient Greek, Roman and early Byzantine writers. Intern Med J 2009; 39: 624-7. [CrossRef]

3. Yantis MA, O'Toole KN, Ring P. Leech therapy. Am J Nurs 2009; 109: 36-42. [CrossRef]

4. Teut M, Warning A. Leeches, phytotherapy and physiotherapy in osteoarthrosis of the knee-a geriatric case study. Forsch Komplementmed 2008; 15: 269-72

5. Munro R, Hechtel FO, Sawyer RT. Sustained bleeding after a leech bite in the apparent absence of hirudin. Thromb Haemost 1989; 61: 366-9.

6. Saha M, Nagi S. Intraperitoneal leech: A rare complication of leech bite. J Indian Assoc Pediat Surg 2011; 16: 155-7. [CrossRef]

7. Ikizceli I, Avsarogulları L, Sozuer E, Yurumez Y, Akdur O. Bleeding due to a medicinal leech bite. Emerg Med J 2005; 22: 458-60. [CrossRef]

8. Etemadi J, Ardalan MR, Motavali R, Tubbs RS, Shoja MM. Thrombotic microangiopathy as a complication of medicinal leech therapy. South Med J 2008; 101: 845-7. [CrossRef]

9. Mory RN, Mindell D, Bloom DA. The leech and the physician: biology, etymology, and medical practice with Hirudinea medicinalis. World $J$ Surg 2000; 24: 878-83. [CrossRef]

10. Fedor PJ. Novel use of a hemostatic dressing in the management of a bleeding leech bite: a case report and review of the literature. Wilderness Environ Med 2012; 23: 44-8. [CrossRef]

11. Zengin $\mathrm{S}$, Yarbil $\mathrm{P}, \mathrm{Kilic} \mathrm{H}, \mathrm{Al}$ B. Prolonged bleeding due to a medicinal leech bite: another treatment method, primary suture. BMJ Case Reports 2012; 13: 1-4. [CrossRef] 